\title{
Probing thermal and acoustic dynamics of inverse silicon metallatices
}

C. Bevis ${ }^{1 *}$, B. Abad ${ }^{1}$, J. Knobloch ${ }^{1}$, T. Frazer ${ }^{1}$, A. Adak ${ }^{1}$, J. Hernández-Charpak ${ }^{1}$, H. Cheng ${ }^{2,3}$, A. Grede $^{3}$, N. Giebink ${ }^{3,5}$, N. Nova ${ }^{2}$, T. Mallouk ${ }^{2,3,6,7}$, P. Mahale, W. Chen ${ }^{3,4}$, Y. Xiong ${ }^{3,4}$, I. Dabo ${ }^{3,4}$, V. Crespi $^{2,3,4,6}$, D. Talreja ${ }^{3}$, H. Kapteyn ${ }^{1}$, V. Gopalan ${ }^{3,4}$, J. Badding $^{2,3,4,6}$, M. Murnane ${ }^{1}$

1. Department of Physics and JILA, University of Colorado and NIST, Boulder, Colorado, USA.

2. Department of Chemistry, Pennsylvania State University, University Park, PA, USA

${ }^{3}$ Materials Research Institute, Pennsylvania State University, University Park, PA, USA

${ }^{4}$ Department of Materials Science and Engineering, Pennsylvania State University, University Park, USA

${ }^{5}$ Department of Electrical Engineering, Pennsylvania State University, University Park, PA, USA

${ }^{6}$ Department of Physics, Pennsylvania State University, University Park, PA, USA

${ }^{7}$ Department of Biochemistry and Molecular Biology, Pennsylvania State University, University Park, PA, USA

*Corresponding author: charles.bevis@colorado.edu

Nanoscale metamaterials are a promising approach to tune the electronic, magnetic, and thermal transport properties of nanosystems which are critically important for next-generation data storage, nanoelectronics, and thermoelectric devices [1]. In particular, inverse metalattices are an effective, bottom-up approach to tune the characteristics of high-frequency phonon transport and interactions, due to precise nanostructuring that is tunable over a broad range of length scales. However, it is still challenging to fully characterize their physical properties in the deep nanoscale regime - since most commonly used optical techniques suffer from the diffraction limit of visible light. Here, we overcome this limit by extending ultrafast extreme ultraviolet (EUV) nanometrology to map the acoustic and thermal transport properties of inverse metalattices.

The metalattices we investigate are made of polycrystalline silicon which is infiltrated into a selfassembled face-centered cubic template of $14 \mathrm{~nm}$ and $30 \mathrm{~nm}$ silica nanospheres. In EUV nanometrology, gratings consisting of periodic nickel nanowires, with periodicity ranging from $120 \mathrm{~nm}-4000 \mathrm{~nm}$ and linewidths from $30 \mathrm{~nm}-1000 \mathrm{~nm}$, are deposited on top of the metalattices. The sample is irradiated with an infrared $25 \mathrm{fs}$ pump pulse to impulsively heat the nanolines, which subsequently thermally expand and launch surface acoustic waves (SAW) into the metalattice and substrate. The grating periodicity controls the SAW wavelength, which is also comparable to the SAW penetration depth. Thus, by reducing the grating period and SAW wavelength, the measurement can therefore be confined to the sample surface, probing only the mechanical properties of the metalattice, independent of the substrate. In addition, our measurements are sensitive to the thermal relaxation of the laser-heated nanogratings, allowing us to observe heat flow through the metalattices. These effects can be simultaneously probed by tracking the change in diffraction efficiency as a function of time delay between the infrared laser pump and a coherent EUV probe, as depicted in Figure 1(a) [2,3]. This probe beam, generated via high harmonic generation, has a $10 \mathrm{fs}$ pulse duration and $30 \mathrm{~nm}$ wavelength which is very well suited to probe the intrinsic time- and length-scales of these nanostructured systems [4].

Through an analysis of the acoustic oscillations observed from the time-resolved data, we extract the SAW velocity as a function of penetration depth through the sample. In addition, we perform finite element analysis (FEA) to calculate the acoustic wave dispersion of the metalattices, which is in excellent 
agreement with experiments for intermediate nanoline sizes. However, deviations from the theoretical value are expected and observed both at large and small penetration depths, as shown in Figure 1(b). At large penetration depths, the acoustic waves partially travel through the silicon substrate affecting the measured SAW velocity. On the other extreme, mass loading starts influencing the measured SAW velocity when the penetration depth becomes on the order to the height of the nanostructures $(\approx 10 \mathrm{~nm})[2]$. These measurements prove that macroscopic predictions effectively predict the mechanical behavior of these nanoscale inverse metalattices since the wavelengths of the probed acoustic waves are still larger than the smaller features of the metalattices. Moreover, the good agreement between theory and experiment indicates both high quality fabrication and measurements of the metalattices.

By comparing the measured thermal decays to FEA, we observed the effective thermal conductivity of these metalattices depends on the heat source, or nanograting, geometry. Additionally, the thermal conductivity value obtained for the small sizes is considerably lower than the predicted one by effective medium theories. Therefore, these results open the door to engineering inverse metalattices for thermoelectric applications - our results corroborate theoretical calculations showing these structures can impede phonon transport while maintaining good electrical conduction [5]. To explain the geometry dependent thermal transport and low thermal conductivity, further characterization of this sample is ongoing through SEM, TEM, and X-ray microscopy studies.

\section{References:}

[1] M Maldovan, , Nature 503 (2013) p. 209.

[2] N Hernandez-Charpak, et al., Nano Lett. 17 (2017) p. 2178.

[3] K Hoogeboom-Pot, et al., Proc. Natl. Acad. Sci. 112 (2015) p. 4846.

[4] A Rundquist, et al., Science 280 (1998) p. 1412.

[5] L Yang, N Yang, and B Li, Nano Lett. 14 (2014) p. 1734-1738.
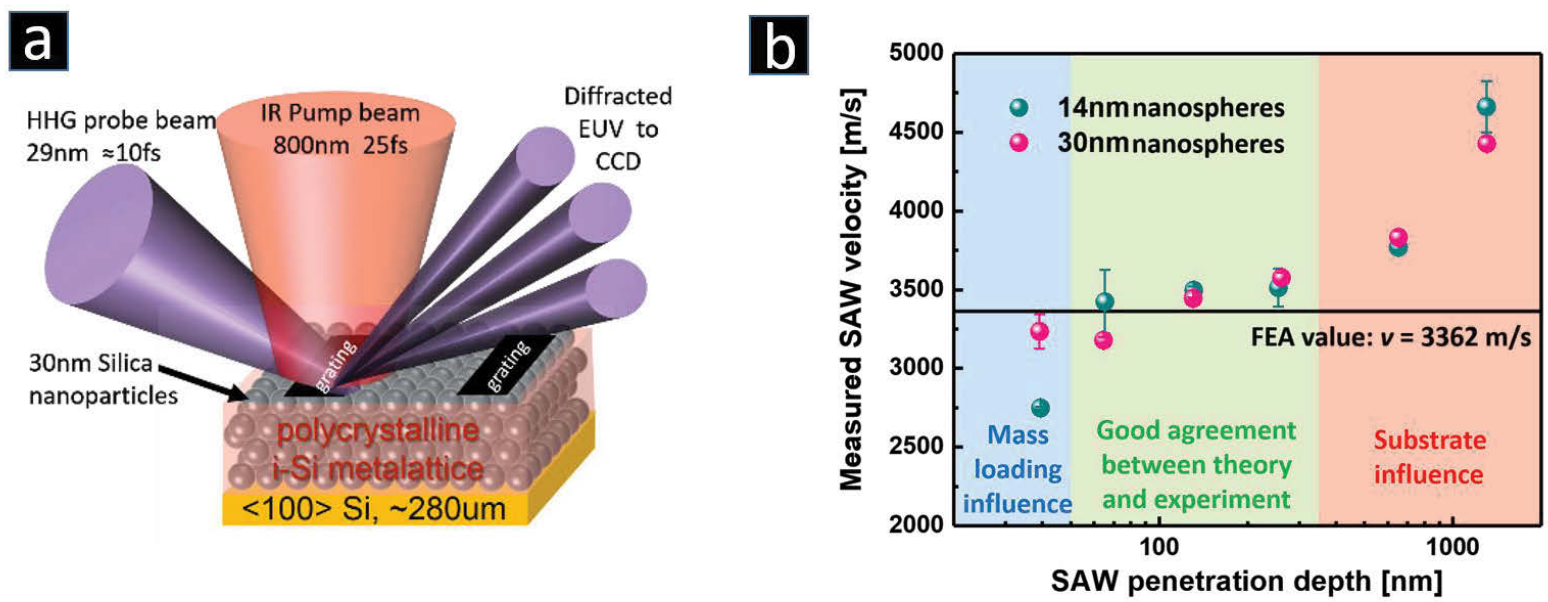

Figure 1. a) A schematic of the experiment. A polycrystalline $\mathrm{Si}$ inverse metalattice is grown on a $\mathrm{Si}$ substrate and Ni nanogratings are fabricated on top. The nanograting is impulsively heated with an infrared pump pulse and sampled with an EUV probe pulse. The diffraction efficiency is studied as a function of delay time between the two pulses. b) SAW velocity as function of penetration depth together with finite element analysis predictions. Both agree well for intermediate penetration depths while deviations are present where substrate and mass loading effects become apparent. 